\title{
Análisis de Calidad de la Energía y Estado de Carga en un Puesto de Seccionamiento y Transformación de Energía
}

\section{Analysis of Energy Quality and Charge Status in a Sectioning Station and Energy Transformation}

DOI: https://doi.org/10.17981/ijmsor.04.01.05

Research Article - Reception Date: Apr 18, 2019- Acceptance Date: Jul 12, 2019

Vladimir Sousa Santos ${ }^{1}$, Julio Gómez Sarduy ${ }^{2}$

Universidad de la Costa CUC. Barranquilla, (Colombia) ${ }^{1}$, Universidad de Cienfuegos (Cuba) ${ }^{2}$

vsousa1@cuc.edu.co,jgomez@ucf.edu.cu

To reference this paper:

V. Sousa Santos, J. Gómez Saurdy "Análisis de calidad de la energía y estado de carga en un puesto de seccionamiento y transformación de energía”, IJMSOR, vol. 4, no. 1, 2019. https://doi. org/10.17981/ijmsor.04.01.05

\begin{abstract}
Resumen-- En este trabajo se presenta la aplicación de un procedimiento para el análisis de calidad de la energía, específicamente de desvío y desbalance de tensión, en un puesto de seccionamiento y transformación de energía (PT), así como se evalúa el estado de carga de sus principales elementos. Para la ejecución del procedimiento no es necesaria la realización de pruebas invasivas o muy costosas. En el trabajo se describen los pasos del procedimiento y se tratan los principales aspectos teóricos aplicados tales como los problemas de la calidad de energía, haciéndose énfasis en el desbalance y desvío de tensión. El procedimiento es aplicado en el (PT) del barrio Torre do Tombo, en la ciudad de Namibe, perteneciente a la Empresa Nacional de Electricidad (ENE) de la República de Angola. Se determinó para cada lectura de tensión y corriente, los parámetros y curvas que permitieron evaluar el estado de carga del transformador, de los disyuntores y de los conductores de cada circuito del (PT), así como realizar un análisis de la calidad de energía de uno de los circuitos, detectándose problemas de calidad de la energía y mala selección de disyuntores.
\end{abstract}

Palabras clave- Calidad de energía; desbalance de tensión; carga de transformador

\begin{abstract}
This work presents the application of a procedure for the analysis of energy quality, specific to voltage deviation and unbalance, in a sectioning and energy transformation (PT) station, as well as evaluating the state of charge of its main elements. In order to carry out the procedure, it is not necessary to carry out invasive or very expensive tests.The work describes the steps of the procedure and deals with the main theoretical aspects applied to stories such as power quality problems, with emphasis on voltage imbalance and deviation. The procedure is applied in the (PT) of the Torre do Tombo neighborhood, in the city of Namibe, belonging to the National Electricity Company (ENE) of the Republic of Angola. For each voltage and current reading, the parameters and curves that allowed evaluating the state of charge of the transformer, the circuit breakers and the conductors of each circuit of the (PT) were determined, as well as performing an analysis of the power quality of one of the circuits, detecting power quality problems and poor selection of circuit breakers.
\end{abstract}

Keywords-- Power quality; voltage imbalance; transformer load 


\section{INTRODUCCIÓN}

La electricidad constituye la forma de energía más usada a nivel mundial, y todo lo que se haga con el objetivo de ahorrarla repercutirá en beneficio general. Diversos estudios de la calidad de energía señalan el desbalance o desvío de tensión, como los problemas más frecuentes encontrados debido a distintas causas, como la presencia de sistemas de potencia relativamente débiles, con transposición incompleta de las líneas, cargas monofásicas, transformadores en conexión abierta, defectos en empalmes, uniones y puentes, entre otros.

Otro factor de creciente preocupación en la ciudad de Namibe, es la selección inadecuada de los elementos que forman parte de un sistema electroenergético, tales como; transformadores, dispositivos de protección, conductores y otros, así como la falta de supervisión durante su funcionamiento, lo que trae consigo fallos, averías y el uso ineficiente de la energía.

En este contexto surge este trabajo, en el cual se aplica un procedimiento para el diagnóstico de problemas de desvío y desbalance de tensión en los sistemas de distribución secundaria, y para evaluar el estado de carga de los principales elementos de un puesto de seccionamiento y transformación de energía (PT) [1].

Para la ejecución del procedimiento no se necesitó realizar pruebas invasivas o muy costosas. Su aplicación tuvo un impacto positivo en el funcionamiento eficiente y económico del puesto de seccionamiento y transformación de energía objeto de estudio en particular, y del sistema de distribución secundario en general.

\section{Desarrollo}

\section{A. Definiciones aplicadas durante el procedimiento}

\section{1) Problemas de calidad de energía}

Los problemas asociados a la calidad de la energía constituyen fenómenos presentes en la vida diaria y consisten en cualquier desviación de la tensión, la corriente o la frecuencia, que provoque la mala operación de los equipos en su uso final y deteriore el confort, la fiabilidad, la disponibilidad o la economía de los usuarios, incrementando el consumo y la demanda y afectando sensiblemente la competitividad empresarial. Su origen puede estar dado en cualquiera de los niveles de un sistema electroenergético, ya sea en transmisión, subtransmisión y distribución, o por particularidades de las cargas en las instalaciones del usuario [2], [3], [4].

En este trabajo se trata el desbalance y desvío de tensión de larga duración.

\section{2) Desvio de tensión de larga duración}

Un sistema eléctrico se encuentra funcionando en condiciones de desvío de tensión de larga duración [5], cuando en intervalos superiores a un minuto, los valores de tensión son diferentes a los de las normas de trabajo establecidas [8].

El valor del desvío de tensión se determina aplicando (1):

$$
\Delta V=\left(\frac{V_{p}}{V_{n}}-1\right) * 100
$$

Donde $\Delta \mathrm{V}$ es la variación de tensión (\%); $V_{\mathrm{n}}$ es la tensión nominal de trabajo $(V)$ y $V_{\mathrm{p}}$ la tensión media $(V)$ calculada por la ecuación (2).

$$
V_{p}=\left(\frac{V_{a b}+V_{b c}+V_{c a}}{3}\right)
$$

Donde $V_{\mathrm{ab}}, V_{\mathrm{bc}}, V_{\mathrm{ca}}$ : constituyen las tensiones de línea (V).

Según el valor de $\Delta V$, el desvio de tensión de larga duración se clasifica en baja tensión o sobre tensión. Se entiende por baja tensión cuando se obtiene un valor de inferior a $0 \%$, por una duración mayor de un minuto [2] y sobre tensión cuando $\Delta \mathrm{V}$ es superior a $0 \%$.

\section{3) Desbalance de tensión}

El desbalance de tensión, que en la mayoría de los casos se presenta junto a desviaciones de tensión, constituye uno de los problemas más comunes y fundamentales de la calidad de la energía, que influye en el incremento de las pérdidas en los sistemas y motores eléctricos.

En el trabajo se calcula el desbalance de tensión utilizando la definición propuesta por la "National Electrical Manufature Association" (NEMA) descrita a continuación [9]:

$$
\begin{aligned}
\operatorname{LVUR}(\%) & =\frac{\operatorname{Max}[|\operatorname{Vab}-\operatorname{Vavg}| ;|\operatorname{Vbc}-\operatorname{Vavgl}| ;|\operatorname{Vca}-\operatorname{Vavgl}|]}{\operatorname{Vavgl}} * 100 \\
\operatorname{Vavgl} l & =\frac{V a b+V b c+V c a}{3}
\end{aligned}
$$

Donde $L V U R(\%)$ es el valor de desbalance de tensión de línea (\%); Max [|Vab-Vavgl|; |Vbc-Vavgl|; |VcaVavgt|] es la máxima desviación de tensión de línea respecto a la tensión promedio $(V)$ y $V_{\text {avgl }}$ es la magnitud de tensión de línea promedio $(V)$.

Para establecer el límite permisible de desbalance de tensión, se utilizó el criterio de la norma NEMA la cual limita su valor a $L V U R \leq 1 \%$ 


\section{4) Puesto de seccionamiento y transformación de energía $(P T)$}

El puesto de seccionamiento y transformación de energía, es la etapa del sistema de distribución en la que se transforma energía desde niveles de media tensión, a niveles de baja tensión para ser utilizados por los usuarios. Estos puntos están compuestos, entre otros elementos, por transformadores, seccionadores, dispositivos de protección, conductores de diferentes calibres y paneles de datos.

Durante la valoración del estado de carga del PT objeto de estudio, se tuvo en cuenta los criterios técnicos que tienen que cumplir sus principales elementos. [3], [4], [5], [7].

\section{B. Procedimiento de trabajo para realizar un análisis de la calidad de energía en el PT y de estado de carga de los principales elementos}

A continuación, se exponen los pasos del procedimiento aplicado.

\section{1) Herramientas necesarias}

Equipo de medición que registre corriente y tensión alterna, computadora y personal con los conocimientos teóricos aplicados en este trabajo y entrenados en el uso de algún programa informático que permita realizar procesamiento de gráficos.

\section{2) Pasos a seguir}

- Obtener los datos nominales del transformador, conductores y disyuntores del PT.

- Realizar las mediciones de tensión y corriente de línea en los circuitos, cada cinco o diez minutos según la característica de carga, y en un período de tiempo considerable que comprenda horarios de máxima demanda de un día típico.

- Procesar los datos, y obtener las curvas del comportamiento en el tiempo del consumo de las corrientes de línea, de las tensiones de línea, del desbalance de tensión (LVUR) y del desvío de tensión. En caso de que LVUR sea superior a 1\%, o que no esté bien definida la tendencia del desvío de tensión, obtener la curva estadística de probabilidad acumulativa.

- Con los valores de corriente obtenidos en el período de máxima demanda, realizar el análisis del estado de carga del transformador, los disyuntores y conductores.

- Interpretar los resultados obtenidos.

- Ejecutar las acciones necesarias si es detectado algún problema.

\section{Caso de estudio}

El análisis de calidad de energía y estado de carga, se realizó en el puesto de seccionamiento y transformación de energía (PT) del barrio Torre do Tombo, en la ciudad del Namibe, Angola. Este PT está compuesto por seis circuitos, cuatro están en funcionamiento y dos circuitos están fuera de servicio. Los circuitos en funcionamiento son denominados: salidas I, II, III y IV respectivamente. A continuación, se muestra los datos nominales de los principales elementos del PT.

En la Tabla 2-1 se muestra los datos nominales del transformador.

TABla 1. Datos nOMinales DEL TRANSFORMAdOR.

\begin{tabular}{|l|l|}
\hline \multicolumn{1}{|c|}{ Fabricante } & \multicolumn{1}{c|}{ Fabricante } \\
\hline Potencia nominal & Potencia nominal \\
\hline Intensidad nominal secundario & Intensidad nominal secundario \\
\hline Conexión & Conexión \\
\hline Tensión nominal primario & Tensión nominal primario \\
\hline Tensión nominal secundario & Tensión nominal secundario \\
\hline Frecuencia & Frecuencia \\
\hline Factor de Potencia & Factor de Potencia \\
\hline Clase de aislamiento & Clase de aislamiento \\
\hline Nivel de Temperatura & Nivel de Temperatura \\
\hline Tensión Pico & Tensión Pico \\
\hline
\end{tabular}

En la Tabla 2 y Tabla 3, se muestran los datos nominales de los disyuntores de cada circuito.

Tabla 2. Datos nominales de los disyuntores de las salidas I, II Y III.

\begin{tabular}{|l|l|}
\hline \multicolumn{1}{|c|}{ Fabricante } & \multicolumn{1}{c|}{ Merlin Gerin } \\
\hline Modelo & compact NS250N \\
\hline Norma & IEC/EN 60947-2 \\
\hline Tensión nominal & $380 / 415 \mathrm{~V}$ \\
\hline Frecuencia & $50 / 60 \mathrm{HZ}$ \\
\hline Corriente nominal & $250 \mathrm{~A}$ \\
\hline Corriente de corto-circuito & $36 \mathrm{kA}$ \\
\hline
\end{tabular}

Tabla 3. Datos nominales del Disyuntor De LA SAlida IV.

\begin{tabular}{|l|l|}
\hline \multicolumn{1}{|c|}{ Fabricante } & \multicolumn{1}{c|}{ Merlin Gerin } \\
\hline Modelo & compact NS250N \\
\hline Norma & IEC/EN 60947-2 \\
\hline Tensión nominal & $380 / 415 \mathrm{~V}$ \\
\hline Frecuencia & $50 / 60 \mathrm{HZ}$ \\
\hline Corriente nominal & $400 \mathrm{~A}$ \\
\hline Corriente de corto-circuito & $50 \mathrm{kA}$ \\
\hline
\end{tabular}


La Tabla 4 representa el calibre de los condutores de cada circuito y la corriente admisible para cada condutor [1].

Tabla 4. Datos de los conductores de cada circuito.

\begin{tabular}{|l|l|l|l|l|}
\hline Salidas & $\begin{array}{c}\text { No. de } \\
\text { conductores }\end{array}$ & Material & $\begin{array}{c}\text { Calibre } \\
\left(\mathrm{mm}^{2}\right)\end{array}$ & $\begin{array}{c}\text { Corriente } \\
\text { admisible (A) }\end{array}$ \\
\hline I,II,III & 4 & Aluminio & 95 & 197 \\
\hline IV & 4 & Aluminio & 240 & 332 \\
\hline
\end{tabular}

\section{1) Magnitudes registradas y procedimiento de medición utilizado.}

Para realizar el análisis de calidad de energía, se midió con intervalos de 10 minutos, la magnitud de tensión y corrientes de líneas en el circuito denominado salida IV, en el período comprendido entre las 7:00 horas hasta las 21:40 de un día típico. Este es un circuito predominantemente residencial, sin embargo, suministra energía a una industria que presenta motores trifásicos de inducción, lo que aumenta la importancia del análisis de la calidad de energía.

Para analizar el estado de carga de los principales elementos del PT, se registraron los valores de corriente en el período desde las 19:20 hasta las 20:00 horas para todos los circuitos. Se seleccionó este intervalo de tiempo, pues está dentro del período de máxima demanda.

Para las mediciones se utilizó un multímetro de marca Clamp Meter DT-266 Digital con precisión del $1.2 \%$ para escala de $750 \mathrm{~V}$ y $3 \%$ para la escala de 1000A. Este multímetro no presenta memoria de registro de datos. Solo fue posible medir la tensión y la corriente pues el equipo no registra otras magnitudes.

\section{2) Análisis de calidad de energía. Interpretación de los resultados}

Para realizar el análisis de calidad de energía se examinó, a partir de los datos obtenidos, el comportamiento del desvío y desbalance de tensión en el circuito "salida IV". Se seleccionó este circuito, pues como se dijo anteriormente, además de alimentar a un sector residencial, suministra energía a una industria con varios motores trifásicos de inducción.

\section{3) Análisis de desbalance de tensión}

El análisis de desbalance de tensión, se hizo a partir de los gráficos de tensión de línea, desbalance de tensión (LVUR) según la norma NEMA, probabilidad acumulativa y comportamiento del consumo de corriente. El valor de LVUR se calculó aplicando las ecuaciones (3) y (4) en cada lectura. La Fig. 1 muestra el comportamiento de las tres tensiones de línea en el tiempo registrado.

Como se puede observar las tres tensiones de líneas tienen diferentes valores efectivos durante todo el tiempo registrado, indicando un posible problema de desbalance de tensión. En la Fig. 2 se presenta el gráfico de desbalance de tensión.

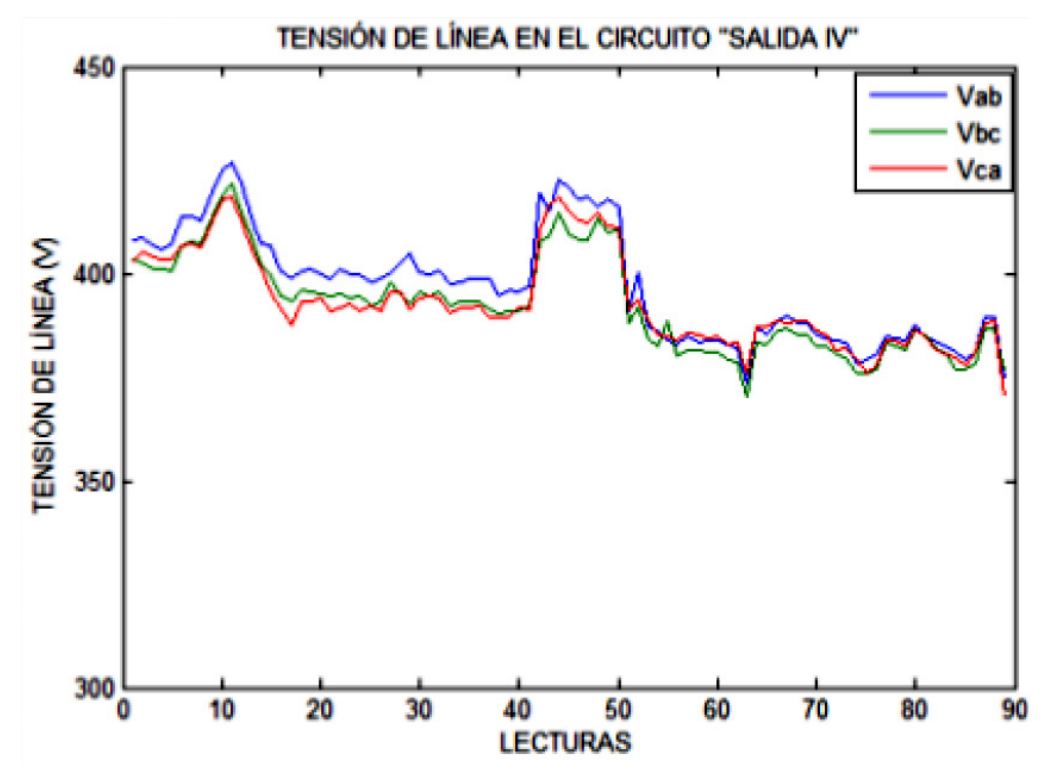

Fig. 1. Comportamiento de la tensión de línea en el tiempo.

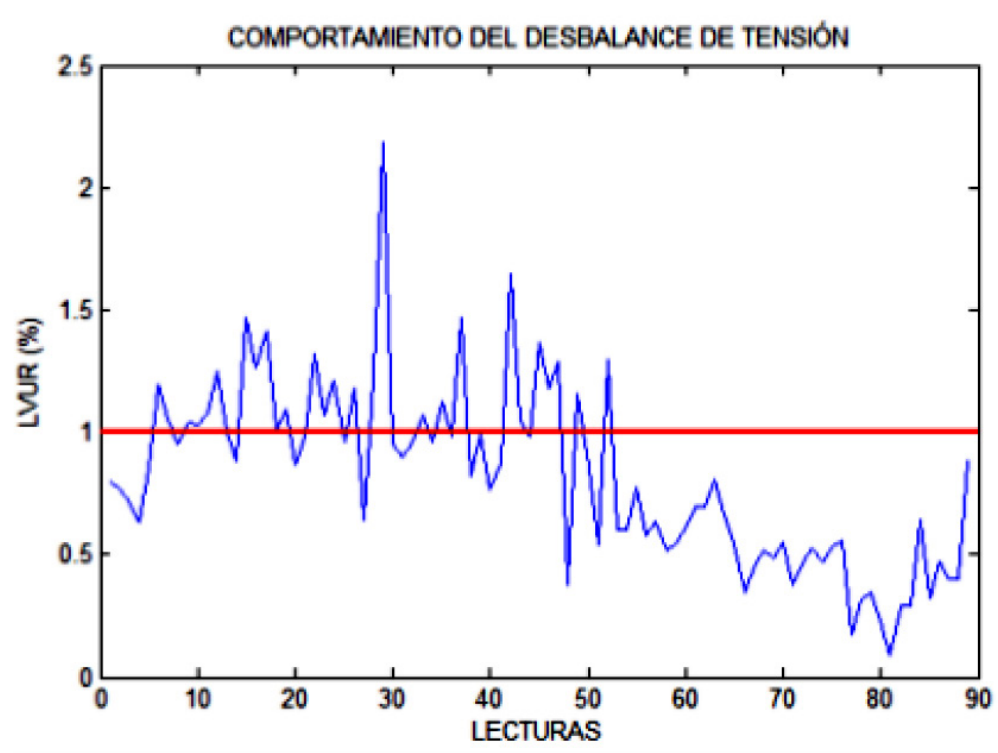

Fig. 2. Comportamiento del (LVUR) en el tiempo.

Como se observa, existen valores superiores a $1 \%$, confirmando de esta forma la presencia de problemas de desbalance de tensión y, por tanto, la posible afectación en los motores asincrónicos que forman parte de este circuito. Es evidente que los problemas de desbalance de tensión ocurrieron desde la lectura 6 a la 52, correspondiente al intervalo de tiempo (7:5015:30), mientras que en el resto de las lecturas (53-89), correspondiente al intervalo de tiempo (15:40-21:40), se manifestó un comportamiento normal. Para definir con mayor precisión la tendencia en el comportamiento de LVUR, se utilizó el gráfico estadístico de probabilidad acumulativa representado en la Fig. 3. Mediante este gráfico se pudo determinar que el $31.5 \%$ de los datos fueron superiores a $1 \%$, es decir, manifestaron problemas de desbalance de tensión. Teniendo en cuenta que una de las causas fundamentales de la 
aparición de problemas de desbalance de tensión es la desigual distribución de las cargas, se procedió al análisis del comportamiento del consumo de corriente en cada fase, lo cual se muestra en la Fig. 4. La figura muestra una considerable desigualdad en la distribución de las cargas, con las fases b y c equilibradas, y la fase a prácticamente sin carga, provocando así el problema de desbalance de tensión señalado, por otro lado, se puede observar el aumento de consumo de corriente a partir de la lectura 73, equivalente a las 19:00 horas, comportamiento normal en un circuito mayoritariamente residencial.



Fig. 3. Gráfico estadístico de probabilidad acumulativa para la variable LVUR.

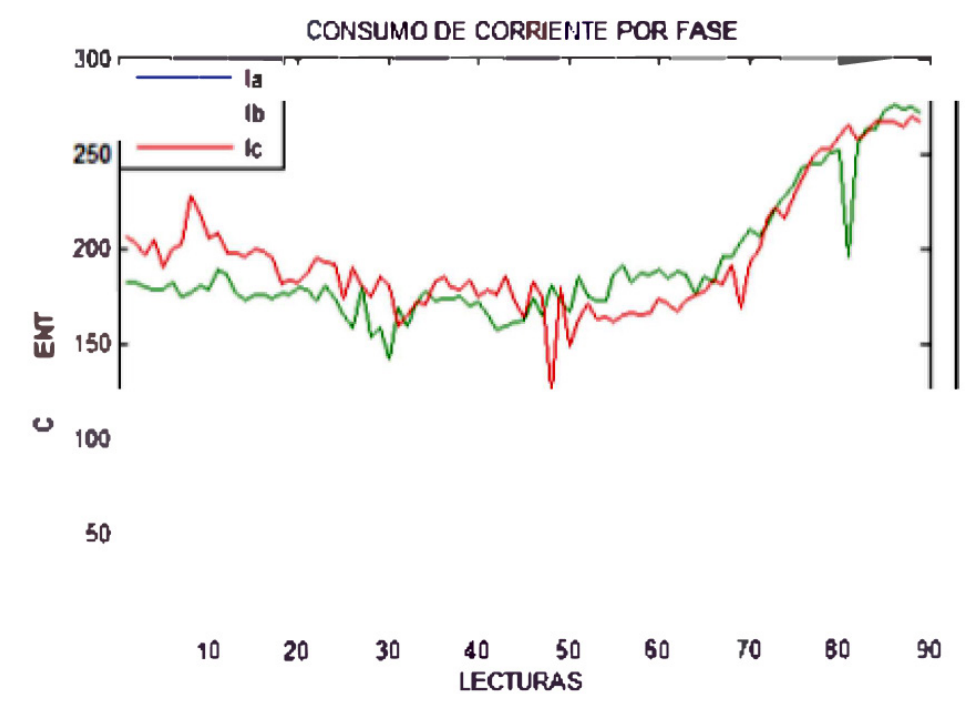

Fig. 4. Comportamiento del consumo de corriente en cada fase.

\section{4) Análisis del desvio de tensión}

Para el análisis del desvío de tensión se aplicó a cada lectura de tensión de línea las ecuaciones (1) y (2). Se seleccionó como tensión nominal de trabajo $400 \mathrm{~V}$, pues este es el valor definido por la ENE como referencia para el sistema de distribución secundaria.

La Fig. 5 representa el comportamiento del desvío de tensión (DT) para todas las lecturas.
COMPORTAMIENTO DE LA DESVIACIÓH DE TENSIÓN EN EL TEMPO

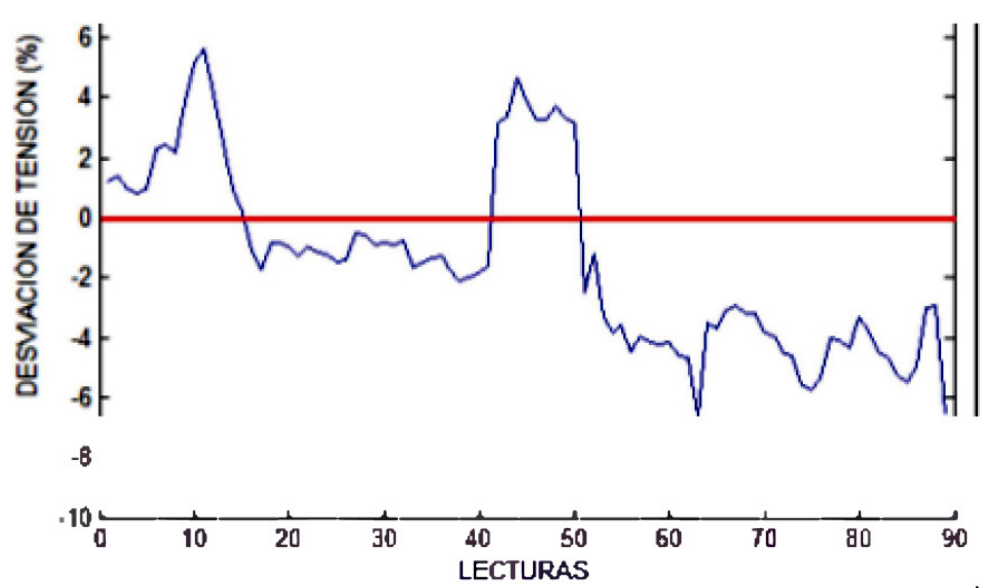

Fig. 5. Comportamiento de la desviación de tensión en el tiempo.

En la Fig. 5 se observa que existen valores de desvío de tensión inferiores y superiores al 0\%, es decir, hay presencia de baja y alta tensión, con un predominio de baja tensión lo cual es preocupante en un PT. No obstante, el valor mínimo de desviación es del -6.668\% y el máximo es del 5.61\%, encontrándose dentro del $\pm 10 \%$, valor estimado como límite en varias normas [2], sin embargo, debe prestarse atención a la baja tensión presente a partir de la lectura 51, equivalente a las 15:20 H.

\section{5) Estado de carga de los principales elementos de PT}

En este epígrafe se analiza el estado de carga del transformador del PT, así como de los disyuntores y conductores de cada circuito. Para esto se utiliza los valores de corriente de línea medidos en cada circuito en la hora comprendida entre las 19:20 horas hasta las 20:00 horas. En la Tabla 5 se muestran estos datos.

\section{6) Transformador}

Para analizar el estado de carga del transformador, se sumó para cada lectura de la Tabla 5, los valores de corriente por fase de todos los circuitos, estos resultados se muestran en la Tabla 6 .

Tabla 5. Valores de corriente total en cada fase

\begin{tabular}{|l|l|l|l|}
\hline \multicolumn{1}{|c|}{ Hora } & $\mathrm{Ia}(1)$ & $\mathrm{Ib}(\mathrm{A})$ & $\mathrm{Ic}(\mathrm{A})$ \\
\hline $19: 20$ & 128 & 420 & 265 \\
\hline $19: 30$ & 118 & 422 & 281 \\
\hline $19: 40$ & 129 & 511 & 300 \\
\hline $19: 50$ & 133 & 459 & 304 \\
\hline
\end{tabular}

Comparando estos resultados con el valor de la corriente nominal de 577.4 A representado en la Tabla 1 , se llega a la conclusión de que el transformador se encuentra trabajando en un estado de carga adecuado, pues en ningún caso se supera el valor de su corriente nominal [6]. 


\section{7) Conductores}

Para analizar el estado de carga de los conductores, se comparó la máxima corriente por fase de cada circuito, con la corriente permisible del conductor correspondiente.

La Tabla 4 muestra que para los conductores de las salidas I, II y III, la corriente permisible es 197 A, valor muy superior a los valores máximos registrados en cada circuito mostrados en la Tabla 5, lo mismo sucede con la salida IV, donde la corriente permisible para el conductor utilizado es 332 A, demostrándose de esta manera, que los conductores se encuentran trabajando en un estado de carga apropiado

\section{8) Disyuntor}

Durante el análisis del funcionamiento de los disyuntores, se pudo detectar que fueron mal seleccionados, pues su corriente de trabajo es superior a la corriente permisible de cada conductor que protege, lo que puede traer como consecuencia que en caso de sobrecarga en los conductores, los disyuntores no accionen, produciendo averías graves en el sistema. Esta situación puede observarse en la Tabla 7, donde se resume los datos de corriente de trabajo de los disyuntores y permisible por los conductores de cada circuito.

\section{Conclusiones}

- El estudio realizado permitió detectar problemas de desbalance y desvío de tensión en el circuito salida IV provocado por la desigual distribución en las cargas. Además de detectar que los disyuntores de protección de los circuitos están mal seleccionados.

- El procedimiento desarrollado es poco invasivo, económico y efectivo para el diagnóstico de problemas de desvío y desbalance de tensión en el sistema de distribución secundaria, y para analizar el estado de carga de los principales elementos de un PT.
- El procedimiento fue evaluado en un caso de estudio, lo que demuestra su viabilidad para las condiciones de Namibe.

\section{REFERENCIAS}

[1] M. Bertolli, G. Roark, S. Urrutia y F. Chiodi, "Revisión de modelos de madurez en la medición del desempeño", INGE CUC, vol. 13, no. 1, pp. 70-83, Jun. 2017. https:// doi.org/10.17981/ingecuc.13.1.2017.07

[2]. CEDAM, "Cables de Alta Seguridad", cedam.com http:// www.cedam.com.ar/IMAGENES/PDF/111)\%20CEDASEG\%20OIL\%20Subterraneo\%20PVC\%201,1\%20 kv\%20Tetrapolar.pdf (visitado Noviembre, 2008).

[3]. J. Duarte, W. Guillín y J. Sánchez, "Desarrollo de una metodología para la predicción del volumen real en la cámara de combustión de motores diésel utilizando elementos finitos", INGE CUC, vol. 14, no. 1, pp. 122-132, Jun. 2018. https://doi.org/10.17981/ingecuc.14.1.2018.11

[4]. Colectivo de Autores. Gestión Energética Empresarial. La Habana, CU: Centro de Estudios de Energía y Medio Ambiente, CEEMA, Universidad de Cienfuegos, 2001.

[5] Cables submarinos, "Conductores Eléctricos", cablessubmarinos-blog.com. http://cablesubmarino.wordpress. com/2008/03/13/conductores-electricos/ (visitado Noviembre, 2008).

[6] Wikipedia, "Disyuntor" Wikipedia.com. http:/l es.wikipedia.org/wiki/Disyuntor (visitado: Diciembre, 2008).

[7] EJM, "Especificação de Disjuntores Segundo a ABNT", ejm.com.br. www.ejm.com.br/download/disjuntores_x_ normas_abnt.pdf (visitado Diciembre, 2008).

[8] A. A. Feodorov y E. Rodríguez. Suministro eléctrico de empresa industriales, La Habana, CU: Pueblo y Educación, 1987.

[9] A. A. Feodorov y E. Rodríguez. Suministro eléctrico de empresa industriales, La Habana, CU: Pueblo y Educación, 1987.

[10] V. Sousa, "Determinación de la eficiencia de motores asincrónicos en condiciones de campo y en presencia de desbalance de tensión", Tesis Master, Fac. Ing., UCF, Cienfuegos, CU, 2006. https://doi.org/10.13140/ RG.2.2.25332.14727

[11] C. Yin Lee, "Effects on Unbalanced Voltage on the operation Performance of a Three Phase Induction Motor", IEEE T Energy Conver, vol 14, no 2, pp. 202-208. Jun. 1999. https://doi.org/10.1109/60.766984

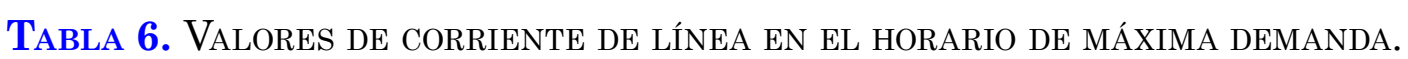

\begin{tabular}{|l|l|l|l|l|l|l|l|l|l|l|l|l|l|l|l|}
\hline \multicolumn{4}{|c|}{ Salida I } & \multicolumn{4}{c|}{ Salida II } & \multicolumn{4}{c|}{ Salida III } & \multicolumn{4}{c|}{ Salida IV } \\
\hline Hora & Ia (A) & Ib (A) & Ic (A) & Hora & Ia (A) & Ib (A) & Ic (A) & Hora & Ia (A) & Ib (A) & Ic (A) & Hora & Ia(A) & Ib(A) & Ic(A) \\
\hline $19: 20$ & 49 & 22 & 21 & $19: 20$ & 37 & 61 & 24 & $19: 20$ & 31 & 145 & 4 & $19: 20$ & 21 & 192 & 216 \\
\hline $19: 30$ & 45 & 33 & 19 & $19: 30$ & 34 & 63 & 22 & $19: 30$ & 36 & 95 & 10 & $19: 30$ & 20 & 231 & 230 \\
\hline $19: 40$ & 48 & 36 & 20 & $19: 40$ & 35 & 66 & 25 & $19: 40$ & 22 & 154 & 13 & $19: 40$ & 26 & 255 & 242 \\
\hline $19: 50$ & 48 & 37 & 18 & $19: 50$ & 34 & 66 & 23 & $19: 50$ & 22 & 82 & 12 & $19: 50$ & 28 & 273 & 260 \\
\hline $20: 00$ & 51 & 38 & 18 & $20: 00$ & 34 & 64 & 20 & $20: 00$ & 32 & 143 & 10 & $20: 00$ & 30 & 274 & 256 \\
\hline
\end{tabular}

TAbla 7. Corriente de trabajo de los disyuntores y PeRmisible Para los CONDUCTORES DE CADA CIRCUITO

\begin{tabular}{|l|l|l|}
\hline \multicolumn{1}{|c|}{ Salidas } & Corriente de trabajo del disyuntor & Corriente admisible por el conductor \\
\hline I, II, III & $250 \mathrm{~A}$ & $197 \mathrm{~A}$ \\
\hline IV & $400 \mathrm{~A}$ & $332 \mathrm{~A}$ \\
\hline
\end{tabular}

